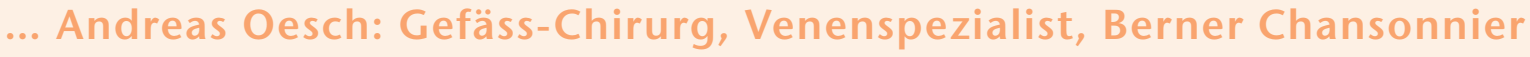 «Es geht um Lebensqualität - auf beiden Seiten»
}

\section{Daniel Lüthi}

Text und Bilder
Wer an die Aussagekraft von Sternbildern glaubt, könnte ihn durchaus für einen Zwilling halten. Typisch, so scheint es, sind dafür die zwei verschiedenen Gesichter, die auf den ersten Blick so gar nicht zusammenpassen wollen: Da ist auf der einen Seite der akribische, pingelig genaue Operateur, Forscher und Dokumentalist, und da ist auf der anderen Seite der unbeschwerte, verspielte, bisweilen skurrile Wortakrobat und Lebenskünstler. Die beiden Gesichter widersprechen sich keineswegs. Aber Andreas Oesch ist ja auch nicht im Zeichen des Zwillings geboren, sondern im Februar. «Am Valentinstag», betont er und lacht.

\section{Das Oesch-Häklein}

Beginnen wir beim Begriff: «Krampfader ist eigentlich eine Verballhornung», erzählt Oesch, «gemeint war ursprünglich die 〈krumbe», die geschlängelte Ader. Aber klar: Es ist natürlich schon ein Krampf, diese Krampfadern rauszunehmen.» Lachen - und die Richtigstellung mit dem treffenden Wort: «zeitaufwendig zumindest».

Der Aufwand lohnt sich, die vielen kleinen farbigen Hefte im Designergestell belegen das. Seit der Eröffnung seiner Privatpraxis 1985 führt Oesch hier ganz genau Buch über jede seiner Varizen-Operatio-

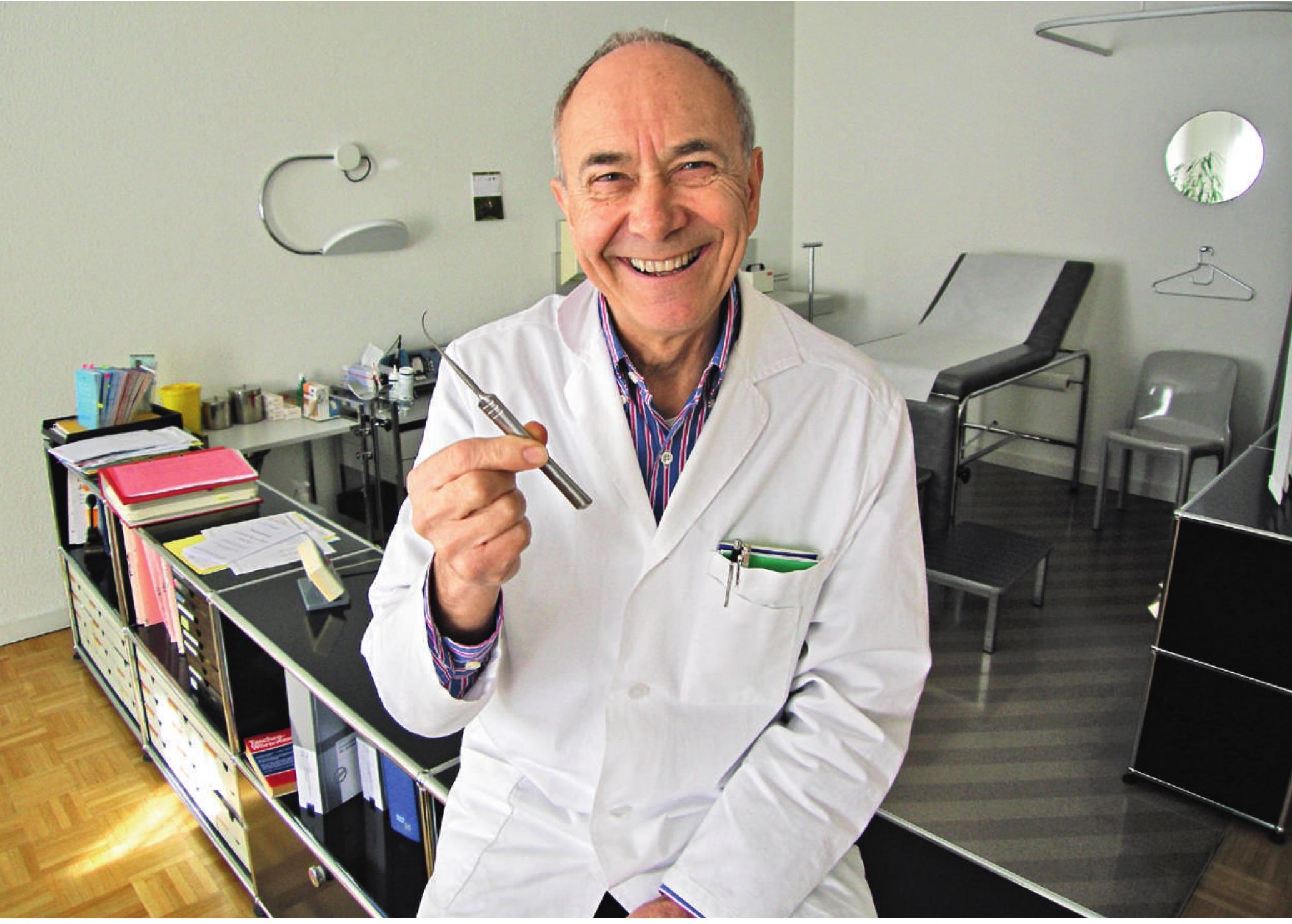


nen, mit einem selber entwickelten Code erfasst er fein säuberlich jeden ausgeführten Schritt, dokumentiert er jede Komplikation. «Möglichst wenig Komplikationen und damit möglichst kurze Arbeitsausfälle sind meine Hauptziele.» Im Laufe der Jahre ist er diesbezüglich immer besser geworden.

Die farbigen Hefte mit ihren genauen Statistiken haben ihm dabei geholfen. Die langjährige Erfahrung und zunehmende Spezialisierung sowieso. Und dazu ein paar wegweisende Kreationen, die seinen Namen tragen, so das berühmte "Oesch-Häklein", das er 1984 entwickelte - auf der Basis eines Instrumentes des Neuenburger Dermatologen Robert Müller, das sich für den Operationssaal kaum eignete. «Das Venenhäkchen ermöglicht die Entfernung von Varizen durch kleine Stichöffnungen von wenigen Millimetern Länge. Diese heute weltweit als selbstverständlich betrachtete Technik war 1984 noch nahezu unbekannt. Die Ära der Krampfadernoperationen mit unzähligen grossen und schmerzhaften Hautschnitten, welche häufig den Heilungsprozess verlängerten, ging damit ihrem Ende entgegen», schreibt in einer Laudatio die Schweizerische Gesellschaft für Phlebologie. Sie ernannte Oesch 2004 zum Ehrenmitglied und schenkte ihm ein silbernes Häkchen. Dieses dekoriert heute das Wartezimmer.

\section{Der PIN-Stripper}

Oesch ist ein Tüftler. Mit dem Erreichten gab er sich nicht zufrieden, forschte und zeichnete weiter. 1992 konnte er eine neue Methode und gleichzeitig ein neues Instrument präsentieren. Während mit dem Oesch-Häkchen nur oberflächliche Krampfadern schonend entfernt werden können, ermöglicht der PIN-Stripper die sanfte Entfernung der wichtigeren, tiefer liegenden Stammvenen. «Der Durchmesser der Strippersonde ist nur noch so gross wie nötig», erklärt Erfinder Oesch, «knapp 2 statt wie bisher bis zu 18 Millimeter, das ist ein grosser Unterschied. Und damit kann auch die erforderliche Hautöffnung auf wenige Millimeter verkürzt werden.» Wieder sucht er nach dem treffenden Wort, überlegt sich wahrscheinlich, ob Eigenlob statthaft ist. Ja, jetzt leuchten seine Augen: «Das war der grosse Durchbruch, darauf bin ich speziell stolz. Der Erfolg kam allerdings nicht über Nacht, dem endgültigen Stripper gingen 17 ständig verbesserte Vorstufen voraus.»

Stolz ist Oesch vor allem auf seine tiefe Komplikationsrate: «0,26 Prozent Thrombosen im ersten Monat, 0,39 Prozent nach zwei Monaten, in den vergangenen drei Jahren keine einzige Thrombose oder Embolie». Eine Bilanz, die Freude macht, denn: «Krampfadern sind kein gravierendes Leiden, da ist jede Komplikation zu viel.»

Apropos Bilanz: Rund 13000 Beine hat Andreas Oesch bisher operiert, 60 Prozent davon im Spital, 40 Prozent ambulant in seiner Praxis. Fast 90 Prozent dieser Beine waren Frauenbeine. «Frauen sind schon ein wenig anfälliger, sie sind aber auch krankheitsbe-

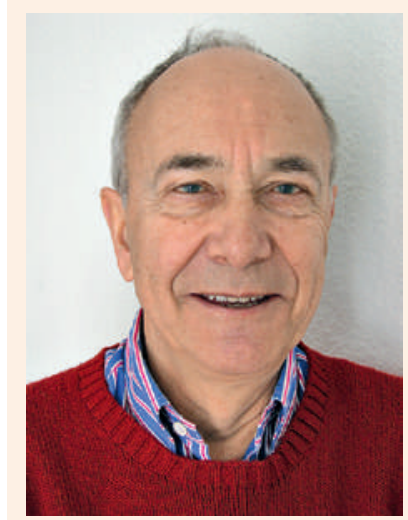

\section{Andreas Oesch}

Dr. med. Andreas Oesch wurde 1945 in Bern geboren, wo er die Schulen besuchte und Medizin studierte. Nach dem Staatsexamen 1970 bildete er sich an der Klinik für Thorax-, Herz- und Gefäss-Chirurgie des Berner Inselspitals zum Gefäss-Chirurgen weiter. Schon bald interessierte er sich für die Spezialität, die auch seinen Vater auszeichnete: die Phlebologie, also das Gebiet der Venenleiden. 1982 bis 1990 leitete er die Venensprechstunde des Berner Frauenspitals. 1984 entwickelte Andreas Oesch ein neues Instrument, das in der ambulanten Phlebektomie weltweit als «Oesch-Häklein» oder «Oesch Hook» bekannt wurde. Anschliessend arbeitete er an einer Verfeinerung des Strippings, also der klassischen Methode zur operativen Therapie von Krampfadern. Dies führte 1993 zur patentierten Technik des PIN-Strippings (PerforationINversion) samt entsprechendem Instrumentarium. Seit 1985 führt Oesch in der Berner Altstadt eine eigene Praxis, in der er ausschliesslich Krampfadern behandelt. Als Belegarzt operiert er Varizen am Berner Salemspital. In den 60er und 70er Jahren gelangte Oesch mit den Berner Trouvères zu nationaler Berühmtheit als Chansonnier.

Andreas Oesch ist Vater von drei erwachsenen Kindern. Er lebt in Bern.

wusster und gehen früher zum Arzt», ergänzt einer, der von sich schalkhaft immer wieder behauptet, er erinnere sich schlecht, vergesse vieles. «Ungefähr um Faktor 1,6, je nach Studie, ist die Anfälligkeit grösser», präzisiert er. Womit wieder der Wissenschaftler spricht.

\section{Die Berner Chansons}

«I vergisse alls» heisst eines der berndeutschen Lieder, die Chansonnier Oesch in den 60er und 70er 
Jahren - sich selber auf einer zwölfsaitigen Gitarre begleitend - in den Kulturkellern Berns und auf den Bühnen dieses Landes sang, gleichzeitig wie Mani Matter beispielsweise, mit dem er gelegentlich einen künstlerischen Austausch pflegte. Das Rendez-vous, den Schirm und den Coiffeur habe er schon vergessen, heisst es darin, und viele andere Sachen, an die er sich nicht mehr erinnere. Der Wortwitz war ihm bei dieser Kreation wohl wichtiger als die Faktentreue. Auch dies ist typisch, so scheint es. For-

\section{«Dieses DRG-System ist doch völlig idiotisch.»}

mulieren und fabulieren gehören eben gleichermassen zu Oesch wie spritzen und strippen. Widersprüchlich ist das nicht, wie gesagt. Es gibt sogar direkte Verbindungen zwischen den beiden Welten, zwischen musikalisch-poetischen Phantasie-Gefilden und medizinisch-chirurgischem Alltag. So widmete Oesch eines seiner humoristisch verspielten Chansons dem Emmentaler Wunderdoktor Micheli Schüpbach, der im 18. Jahrhundert bei einem Patienten «Gastrobelzebubsatanitis» diagnostizierte, ihm die Teufel im Bauch mit einer gehörigen Ladung Strom austrieb und die dafür kassierten Goldstücke den Armen weiterverschenkte. Moral der Geschichte: «Eso ischs no ggange vor zwöihundert Jahr - hüt geit es nümm eso, nid wahr.»

Eine weitere Brücke zwischen den beiden Welten ist die: Derjenige, der neben Oesch seinerzeit auf der Bühne stand und ihn am Bass begleitete, steht seit 26 Jahren regelmässig neben ihm am Operationstisch und begleitet mit verschiedenen Instrumenten noch heute seine Krampfadern-Behandlungen. Auch der Bassist Christian Fischer ist Chirurg.

Bei den Berner Trouvères war Andreas Oesch neben dem tiefsinnigen Peter Krähenbühl oder dem philosophischen Werner Jundt beispielweise - mehr der locker flockige Humorist, der sich über politischen Protest eher lustig machte als sich bei derlei Themen in Rage zu singen. Anders heute in der Arztpraxis.

\section{Kostentreiber DRG}

Angesprochen auf politische Komponenten seiner Tätigkeit im Spital, wird er plötzlich ernst, ja bissig:
«Dieses DRG-System ist doch völlig idiotisch. Es nimmt einem Arzt beispielsweise jeden Anreiz, bei einer Operation unter derselben Narkose gleich zwei Dinge zu operieren, also zwei Probleme gleichzeitig zu lösen. Die 2. Operation wird von der Krankenkasse nämlich nur zu 50\% vergütet, da hospitalisieren viele Ärzte einen Patienten lieber zweimal. Zweites Beispiel: DRG sieht vor, dass ich eine Krampfadern-Patientin zwei Nächte lang im Spital behalte, dabei reicht eine Nacht in aller Regel völlig. Oder: Ein Krankenkassenvertreter sagt mir vom Schreibtisch aus, was ich ambulant operieren soll und was nicht. Eigenartigerweise wird bei einer ambulanten Operation gleichzeitig auch die ärztliche Assistenz gestrichen. Dies kann natürlich Einbussen bei der Qualität zur Folge haben. Konkret bedeutet dies alles: Bürokraten sorgen zunehmend dafür, dass die Kosten im Gesundheitswesen steigen.»

\section{Der Genussmensch}

Und während für Chirurgen der Zeitdruck steigt, sagt Routinier Oesch gelassen: «Ich habe mich nie stressen lassen, ich nehme mir die Zeit, die ich brauche. Denn: Es geht um Lebensqualität - auf beiden Seiten. Ich will die Lebensqualität meiner Patientinnen und Patienten erhalten oder verbessern, gleichzeitig aber auch meine eigene nicht vernachlässigen.» Konkret heisst das beispielsweise: «Ich mache höchstens drei Operationen pro Tag.» Oder: «Ich empfehle nur, was ich auch bei mir selber machen lassen würde.»

Insgesamt heisst das aber auch, dass bei Andreas Oesch zum Grübeln der Genuss gehört. Und dass nach der Arbeit eben das Vergnügen kommt: der regelmässige Ausflug mit Freunden nach Italien beispielsweise, «wo wir reichlich essen, trinken und Sprüche klopfen». Oder die einsamen Sessions zu Hause am Keyboard, «wo ich schrecklich kitschige Melodien spiele - mit Kopfhörer selbstverständlich, damit niemand in der Umgebung zu Schaden kommt».

Dazu gehört auch die Freude an den Formen und der Kraft von alten Automobilen. Symbolisch dafür stehen im Schrank neben der weissen und der blauen Arztschürze ein paar schöne Modell-Versionen, darunter ein edler Maserati. Symbolisch auch die Aussage dazu: «Technik, Form und Funktion gehören zusammen - wie bei meinen Instrumenten auch.» Wieder leuchten die Augen dieses Arztes, der nicht nur Arzt ist. Und wieder wird klar, dass bei ihm die beiden Seiten, die an das Sternzeichen Zwilling erinnern, untrennbar zusammengehören.

\section{Die nächste «Begegnung mit ...»}

Am Ende jeden Monats stellt die Schweizerische Ärztezeitung eine Persönlichkeit vor, die sich im Gesundheitswesen engagiert. Im Mai schildert Daniel Lüthi seine Begegnung mit Katrin Raess, Fachärztin für Urologie (1998 die erste in der Schweiz). 